\title{
How Did Radiologists' Diagnostic Accuracy Have Changed in Covid-19 Pneumonia; a Single Centre Retrospective Study
}

\author{
Ali Murat Koc ${ }^{1}$, Levent Altın${ }^{1}$, Türker Acar ${ }^{1}$, Alpay Arı ${ }^{1}$, and Zehra Hilal Adıbelli ${ }^{1}$ \\ ${ }^{1}$ University of Health Sciences Izmir Bozyaka Education and Research Hospital
}

February 1, 2021

\begin{abstract}
Aims Delay and false positivity in PCR test results have necessitated accurate chest CT reporting for management of patients with COVID-19 suspected symptoms. Pandemic related workload and level of experience on covid-dedicated chest CT scans might have effected diagnostic performance of on-call radiologists. The aim of this study is to reveal the interpretation errors in chest-CT reports of COVID-19 suspected patients admitted to the ER. Methods COVID-19 dedicated chest-CT scans which were performed between March and June 2020 were re-evaluated and compared with the former reports of these scans and PCR test results. CT scan results were classified into four groups. Parenchymal involvement ratios, radiology departments' workload, COVID-19 related educational activities have examined. Results Out of 5721 Chest-CT scans, 783 CTs belonging to 664 patients (340 female, 324 male) were included to this study. RT-PCR test was positive in 398; negative in 385 cases. PCR positivity was found to be highest in "normal" and "typical for covid" groups whereas lowest in "atypical for covid" and "not covid" groups. 5-25\% parenchymal involvement ratio was found in $84.2 \%$ of the cases. Regarding number of chest CT scans performed, radiologists' workload have found to be increased six-folds compared to the same months of the former year. With the re-evaluation, a total of 145 IEs (18.5\%) have been found. IEs were mostly precipitated in the first two months (88.3\%) and mostly in "not covid" class $(60 \%)$ regardless of PCR positivity. COVID-19 and radiology entitled educational activities along with the ER admission rates within the first two months of pandemic have seem to be related with the decline of IEs within time. Conclusion COVID-19 pandemic made a great impact on radiology departments with an inevitable burden of daily chest-CT reporting. This workload and concomitant factors have possible effects on diagnostic challenges in COVID-19 pneumonia.
\end{abstract} How Did Radiologists' Diagnostic Accuracy Have Changed in Covid-19 Pneumonia; a Single
Centre Retrospective Study

\section{ABSTRACT}

\section{Aims}

Delay and false positivity in PCR test results have necessitated accurate chest CT reporting for management of patients with COVID-19 suspected symptoms. Pandemic related workload and level of experience on coviddedicated chest CT scans might have effected diagnostic performance of on-call radiologists. The aim of this study is to reveal the interpretation errors in chest-CT reports of COVID-19 suspected patients admitted to the ER.

\section{Methods}

COVID-19 dedicated chest-CT scans which were performed between March and June 2020 were re-evaluated and compared with the former reports of these scans and PCR test results. CT scan results were classified into four groups. Parenchymal involvement ratios, radiology departments' workload, COVID-19 related educational activities have examined. 


\section{Results}

Out of 5721 Chest-CT scans, 783 CTs belonging to 664 patients (340 female, 324 male) were included to this study. RT-PCR test was positive in 398; negative in 385 cases. PCR positivity was found to be highest in "normal" and "typical for covid" groups whereas lowest in "atypical for covid" and "not covid" groups. $5-25 \%$ parenchymal involvement ratio was found in $84.2 \%$ of the cases. Regarding number of chest CT scans performed, radiologists' workload have found to be increased six-folds compared to the same months of the former year. With the re-evaluation, a total of 145 IEs (18.5\%) have been found. IEs were mostly precipitated in the first two months (88.3\%) and mostly in "not covid" class (60\%) regardless of PCR positivity. COVID19 and radiology entitled educational activities along with the ER admission rates within the first two months of pandemic have seem to be related with the decline of IEs within time.

\section{Conclusion}

COVID-19 pandemic made a great impact on radiology departments with an inevitable burden of daily chest-CT reporting. This workload and concomitant factors have possible effects on diagnostic challenges in COVID-19 pneumonia.

\section{Keywords:}

COVID-19, radiology, Chest, CT scan, diagnostic, accuracy

\section{Bulletins:}

- The crucial role of radiologists specifically their interpretation in chest CT scans in diagnosis of COVID19 pneumonia have better understood with the spread of pandemic.

- Pandemic related workload and level of experience on covid-dedicated chest CT scans have effected diagnostic performance of on-call radiologists.

- This study have depicts the rate and possible causes of interpretation errors in chest-CT reports of COVID-19 suspected patients admitted to the ER.

\section{INTRODUCTION}

COVID-19 pneumonia was detected for the first time in the world in Wuhan, China in 2019 and has spread all over the world over time. This virus, SARS-CoV-2, enters the body and causes from an asymptomatic course to a severe pneumonia with the need for intubation probably depending on the strength of the immune system, viral load, age of the patient and comorbid diseases. Hence the virus can lead to a wide spectrum of findings, additional testing is a must to make the correct diagnosis. The sensitivity of the real-time reverse transcription-polymerase chain reaction (RT-PCR) tests used for diagnosis is variable and the use of Computed Tomography (CT) is very important for diagnosis in cases with pneumonia. (1-3) As the number of cases increased all over the world, CT findings of COVID 19 pneumonia have become better recognized and the scope of findings has expanded.(4) Total number of cases worldwide has reached 62 million by October, 2020 (5). Since the March 10, 2020; when the first case was seen in our country, CT examination was routinely performed to patients with suspicion of covid pneumonia together with the PCR testing. We think that our experience of CT findings in COVID-19 pneumonia was increased with the increasing number of cases in this timeframe. Besides, there may have been differences in the evaluation of CT examinations due to the increasing workload in the days when the number of admission was high.

In this study, it was aimed to find out the possible changes in the awareness and knowledge level of the radiology department by retrospectively re-evaluating the reports of the chest computed tomography images of patients who admitted to the emergency room with the suspicion of COVID-19.

\section{METHODS and MATERIALS}

\section{Patient Selection}

Chest Computed Tomography of patients who admitted to our hospital's emergency department with suspicion of COVID-19 between March and June 2020 were enrolled. Among these, patients who have RT-PCR 
tests performed via nasopharyngeal and oropharyngeal swap specimens were selected. PCR tests had been finalized within 24 to 96 hours at the first days of the epidemic in our country. Patients whose CT scans performed with a time gap longer than 48 hours with PCR testing were excluded from the study.

Ethics committee approval was received for this study both from the institutional Ethical Committee and The Ministry of Health.

\section{CT acquisition technique}

Radiological assessment of patients included unenhanced Chest CT imaging with covid-dedicated scanning protocols in two scanners (128-MDCT Siemens Somatom Definition; 16-MDCT Toshiba Alexion): supine, end-inspiration acquisition; slice thickness, 1.0-1.5 mm; tube voltage, $120 \mathrm{kV}$; tube current, 200-300 mAs; multiplanary reconstructions with mediastinal and lung parenchymal window settings.

\section{Radiological evaluation}

Computed Tomography images were reretrospectively re-evaluated by 3 radiologists. Typical and atypical chest CT findings related to Covid 19 pneumonia were evaluated separately by each radiologist blinded to the previously written reports. Multifocal ground glass opacities (GGO), consolidation, GGO with superimposed consolidation, consolidation predominant pattern, crazy paving pattern, and melted sugar sign were considered as typical; pleural and/or pericardial effusion, cavity, pulmonary nodule, nodular pattern, lymphadenopathy, peribronchovascular distribution, halo and/or reverse halo sign, three-in-bud sign, bronchiectasis, airway secretions, pulmonary emphysema, pulmonary fibrosis, isolated pleural thickening, and pneumothorax were considered as atypical findings for covid pneumonia.(6) Patients were categorised as "normal", "typical for covid", "atypical for covid" and "not covid" similarly with the previous structured reports. Afterwards, parenchymal involvement ratios were visually defined for "typical for covid" and "atypical for covid" groups; $<25 \%, 26-50 \%, 51-75 \%$, and $76-100 \%$. In case of a conflict between evaluations, the decision was made with the consensus of three radiologists. In addition to this re-evaluation, a forth radiologist searched for preliminary reports, final reports and radiology consultation notes of these patients. The point of this thoughtful research is to find out the very first on-call radiologist's comment on CT images.

Results of the re-evaluation were compared with previously written reports and also with PCR test results. By this way, the diagnostic accuracy of $\mathrm{CT}$ reports was aimed to be determined throughout the whole process from the first stage of the epidemic to the present.

\section{Workload and Educational Activities}

The number of Thoracic CTs acquired for each 24-hour working period was recorded to point out the possible diagnostic differences on the high intensity workdays. Also, total number of reported COVID-19 pneumonia suspected CTs were cumulatively calculated to find out the break-point of departmental learning curve in accurate diagnosis. Schedules of in-department teaching activities, face-to-face meetings, and online meetings, conferences, webinars organized by the National Radiology Association were also noted. The possible positive effects levering the quality of CT reporting for covid-pneumonia were investigated.

Statistical Analysis: (örnek)

Statistical analyses were performed using the SPSS software version 22. Distribution of clinical characteristics across groups of CT and RT-PCR results were presented with frequency tables. The Chi-square test was used to compare these proportions in different groups. The Chi-square test or Fisher's exact test was performed to test the significance of pairwise differences using Bonferroni correction to adjust for multiple comparisons. An overall \%5 type-I error level was used to infer statistical significance.

\section{RESULTS}

In this cohort; a total of 5721 chest-CT scans were found to have acquired at our institution during March, April and May 2020. Chest CT's were mostly requested in patients with symptoms of persistent cough, fever, history of pulmonary nodule, mass, chest operation, trauma etc. 1478 patients were removed from the study 
due to the long latency of PCR results. 3460 patients who didn't have PCR test at our institution were also removed. Remaining 783 Chest CT scans of 664 patients who have PCR test results were retrospectively included (Figure 1). 82 patients had multiple CT scans during their hospitalization period. 340 patients were female $(51.2 \%)$ and 324 patients were male $(48.8 \%)$. RT-PCR test was positive in 398; negative in 385 cases.

\section{Radiological evaluation:}

A total of 783 chest scans were re-evaluated with three separate radiologists blinded to the former CT scan reports and PCR test results. Parenchymal involvement analysis were executed via visual semi-quantitative analysis of the three radiologists. Significant amount of CT scans resulted in only mild $(<25 \%)$ parenchymal involvement $(\mathrm{n}=405,84.2 \%) .57$ patients showed $25-50 \%$ parenchymal involvement (11.85\%). Only 19 patients showed higher percentage $(>50 \%)$ of parenchymal involvement $(3.95 \%)$ (Table 1$)$.

A total of $132 \mathrm{CT}$ scan were classified as "normal" whereas $170 \mathrm{CT}$ scans as "not covid". PCR test results were positive in nearly all of the "normal" group $(\mathrm{n}=121,91.7 \%)$; whereas negative in most of the "not-covid" group $(\mathrm{n}=143,84.1 \%)$. Remaining $481 \mathrm{CT}$ scans were suspicious for COVID-19 pneumonia. PCR test results were positive in $59.2 \%$ of "typical for covid" classified scans, while negative in $70.3 \%$ of "atypical for covid" ones. Distribution of PCR test results among results of CT scans have shown a statistically significant difference $(\mathrm{p}<0,001)$. (Table 2).

Results of re-evaluation were compared with former documents related to the CT scans. It has been found that, radiological evaluations of a total of $145 \mathrm{CT}$ scans (18.5\%) have changed owing to the increased knowledge and experience in time. After this point, these diagnostic differences will be referred as "interpretation errors" (IE). Retrospective evaluation was performed six months after the first phase of the pandemic (first three months) in our country. Number of CT scans, interpretation errors, and their monthly distribution had calculated (Figure 2). Afterwards, results of CT scans according to the aforementioned four classifications (normal, covid, typical for covid, atypical for covid and not covid) were correlated with the number of interpretation errors and PCR test results. Distribution of IEs among results of CT scans have shown a statistically significant difference $(\mathrm{p}<0,001)$. (Table 2$)$. Furthermore, it has been found that IEs were mostly precipitated in "not covid" group regardless of PCR positivity $(\mathrm{n}=87,60 \%)$. "Atypical for covid" group was found to be the second most common one that IEs have determined $(\mathrm{n}=44,30.34 \%)$. Ratio of IEs in "normal" and "typical for covid" groups constitute less than ten percent of all $(9.66 \%)$. Findings were summarized at table 3 .

\section{Workload Volume and Educational Activities:}

Radiologists' workload volume especially in on-call hours vary mostly depending on the ER admission rates. At our institution, primarily one radiologist at night shifts and one radiologist at working hours were responsible for emergency cases. The total number of chest-CT scans reported have markedly increased up to 140 cases/day.

Educational activities dedicated to the novel-coronavirus infection that took place within the radiology department and also within institutional panels, symposiums organized by national radiological society, ministry of health and online meetings have been investigated with their dates (appendix 1). It has been found that local/national educational activities mostly aggregated at the end of March and the first half of April.

Results of re-evaluation of chest CT scans were correlated with former documents and gold standard PCR test results. IEs were correlated with workload and educational activities. By this way, the learning curve in radiological diagnosis of COVID-19 pneumonia was tried to be established. It has been found that, the number of chest-CT scans increase to the top level at the beginning of April than gradually decrease. On the other hand, the distribution of the ratio of IEs to the number of chest-CT scans among this three month period have pointed out that the ratio of IEs gradually decrease more than half after April, from $20.2 \%$ to $11.18 \%$ (figure 1).

\section{DISCUSSION:}


In our study, we aimed to expose the radiologists' increasing experience on evaluation of chest-CT findings of COVID-19 pneumonia. We have focused on some unobserved challenges on radiologists' in the COVID-19 pandemic to better understand the diagnostic differences in re-evaluation.

Correlation of re-evaluated chest CT results with PCR tests have demonstrated that PCR test was negative in $84.1 \%$ of the scans in "not covid" group. On the other hand, the $91.7 \%$ PCR-positivity were calculated in the cases labelled for "normal". This finding can be explained with the vigorous use of CT in the ER setting, even for the patients who has COVID-19 infection but lack of pneumonia. Calculations with "typical for covid", "atypical for covid" and combination of this two groups have resulted in $62.8 \%$ sensitivity and $40 \%$ specificity of Chest-CT according to PCR test results.

We have identified diagnostic differences in $18.5 \%$ of the chest CT scans in our cohort. The distribution of this EIs have shown a dramatic accumulation within the first 60 days of pandemic in our country. After this point, the of IEs decrease down to $11.18 \%$ (figure 2). There are some reasons that can explain the possible causes of this relatively high EI ratios and its course among the first three months of pandemic. First of all, both national and global total number of cases and new confirmed cases prominently increased within the first two months. The number of total cases significantly increase from $\sim 116.000$ to $\sim 3.2$ million with an increase in new confirmed case from 4600 to 86.000 /day worldwide. Similarly, a total of $\sim 120.000$ cases have been identified in our country between 10th March and $1^{\text {st }}$ May, 2020 with a 2615 /day new confirmed cases on $1^{\text {st }}$ May (5). Our institution as well as most of the healthcare centres worldwide have faced striking hospital admission rates up to $1000 /$ day with the spread of coronavirus. Between $1^{\text {st }}$ of May and $1^{\text {st }}$ of June, new confirmed daily cases decrease by $67.92 \%$. As of $8^{\text {th }}$ of January 2021, the cumulative number of covid cases are nearly 1.5 million in our country (5). We have re-evaluated the study cohort with a remarkable experience of $\sim 17.000$ chest CT scans reported in the past 9 months at our institution.

During pandemic, workload of radiologists have expeditiously increased parallel to the admissions with the suspicion of COVID-19. According to the COVID-19 guidelines published by the ministry of health (appendix-2), chest CT scanning have become the secondary diagnostic tool after the gold standard PCR testing. To overcome the delay in diagnosis due to the PCR testing, clinicians encouraged to use the CT scan as the quickest way to isolate/hospitalize the COVID-19 infected patients (7). Moreover, Chest-CT gained an important role in clarification of PCR-false negative but clinically highly suspected cases.(8) These all have seem to be contributed to acquisition of 5721 Chest CT-scans within this three month period. For comparison, there were approximately 900 chest CTs performed at out institution between the same months of the last year (March to June, 2019). Radiologists on shifts were directly affected from this six-fold increase; up to a total of 140 covid-suspected chest CT scans were reported in a day, along with the other non-covid emergencies. Reporting time was approximately 10-30 minutes after the acquisition. Another difficulty was that the covid dedicated CT scanning protocol included approximately 320 axial slices with a $1 \mathrm{~mm}$ slice thickness which makes it more time consuming than the standard chest CT scanning protocol with 3-5 mm slice thickness. All in all, exponentially increased admission rates along with the increased number of covid dedicated, thin slice chest CT scans have become grounds for possible interpretation errors.

Another point worth mentioning is that; although most typical signs of covid pneumonia on chest CT had first identified in Wuhan-China, numerous atypical signs had evolved with the spread of the infection to different profile of patients. As Falaschi et al . stated, incorporation of China experience along with the other previously contaminated countries into daily clinical practice seems to have caused an improvement in our diagnostic accuracy (4). The recognition of the relationship between atypical findings for COVID-19 pneumonia on Chest CT may have contributed to the decline of IE rate. Another possible contributor is the aggregation of educational activities focused on radiological findings of covid pneumonia in the first two months.

Further analysis have shown a marked uneven distribution of IEs among the four CT result groups. More than half of the errors have been made in the "not covid", followed by the "atypical for covid" group. IEs in the re-evaluated "not covid" group were mostly consisted of incorrect diagnosis for "atypical for covid". Similarly, analysis of re-evaluated "atypical covid" group have shown a marked incorrect diagnosis for 
"typical for covid". These findings have shown us that that the radiological hallmarks of covid pneumonia were successfully identified but there were lack of experience and knowledge, specifically about atypical radiological findings. This situation had probably created a tendency to not to exclude the covid diagnosis, yet point out a suspicion with the statement of "atypical for covid" in CT reports. Another issue to mention is that, there are subspecialized academic and staff radiologists at our department. The COVID-19 pandemic have necessitated rapid responses from radiology departments to overcome the increased demand on diagnosis and management of covid pneumonia. Along with the partial cancellation of elective diagnostic/interventional procedures and outpatient diagnostic services; nearly all radiologists were integrated into a team dedicated to COVID-19 diagnosis. Therefore, as Vijayasarathi et al . stated, subspecialised radiologists have faced an unexpected challenge in this crisis to maintain their versatility in reporting chest CT scans (9). Similarly, Shi et al. and Cavallo et al. have also mentioned imperative outside-role definition of radiology employees $(10,11)$. Hereby, this situation might also contribute to the escalation of interpretation errors. Considering together with the increased workload and physical and psychological stress of pandemic, it seems nearly inevitable to make interpretation errors in the COVID-19 outbreak.

There are some limitations this study. First, we could have included only $13.6 \%$ of the Chest CT scans due to lack or delay of PCR test results. Second, we have ignored decline in non-COVID CT cases per/day and focused on COVID dedicated CT reporting volume.

There's no doubt that COVID pandemic made a huge impact on radiology practices worldwide. Increment of covid-related admission rates and relevant increase in daily workload, rotations of subspecialized personnel for urgent health care management along with growing diagnostic experience over time have possible effects on radiology departments' performance.

\section{REFERENCES}

1. Kwee TC, Kwee RM. Chest ct in COVID-19: What the radiologist needs to know. Radiographics. 2020;40(7):1848-65.

2. Duarte ML, Santos LR Dos, Contenças AC de S, Iared W, Peccin MS, Atallah ÁN. Reverse-transcriptase polymerase chain reaction versus chest computed tomography for detecting early symptoms of COVID-19. A diagnostic accuracy systematic review and meta-analysis. Sao Paulo Med J. 2020;138(5):422-32.

3. He J-L, Luo L, Luo Z-D, Lyu J-X, Ng M-Y, Shen X-P, et al. Diagnostic performance between CT and initial real-time RT-PCR for clinically suspected 2019 coronavirus disease (COVID-19) patients outside Wuhan, China. Respir Med [Internet]. 2020/04/21. 2020 Jul;168:105980. Available from: https://pubmed.ncbi.nlm.nih.gov/32364959

4. Falaschi Z, Danna PSC, Arioli R, Pasché A, Zagaria D, Percivale I, et al. Chest CT accuracy in diagnosing COVID-19 during the peak of the Italian epidemic: A retrospective correlation with RT-PCR testing and analysis of discordant cases. Eur J Radiol. 2020 Sep;130:109192.

5. WHO Coronavirus Disease (COVID-19) Dashboard [Internet]. 2020 [cited 2021 Jan 9]. Available from: https://covid19.who.int/

6. Salehi S, Abedi A, Balakrishnan S, Gholamrezanezhad A. Coronavirus disease 2019 (COVID-19) imaging reporting and data system (COVID-RADS) and common lexicon: a proposal based on the imaging data of 37 studies. Eur Radiol. 2020;30(9):4930-42.

7. Fang Y, Pang P. CT VS RTPCR sensitivity. 2020;1-3.

8. Tavare AN, Braddy A, Brill S, Jarvis H, Sivaramakrishnan A, Barnett J, et al. Managing high clinical suspicion COVID-19 inpatients with negative RT-PCR: A pragmatic and limited role for thoracic CT. Thorax. $2020 ; 537-8$.

9. Vijayasarathi A, Khosa F. Learning from COVID-19: Sub-specialty radiologists should maintain general radiology skillset. Clin Imaging. 2020 Nov;67:192-3. 
10. Cavallo JJ, Forman HP. The economic impact of the COVID-19 pandemic on radiology practices. Radiology. 2020;296(3):E141-4.

11. Shi J, Giess CS, Martin T, Lemaire KA, Curley PJ, Bay C, et al. Radiology Workload Changes During the COVID-19 Pandemic: Implications for Staff Redeployment. Acad Radiol [Internet]. 2020;28(1):1-7. Available from: https://doi.org/10.1016/j.acra.2020.09.008

\section{FIGURE LEGENDS}

Figure 1. Study flow-chart

Figure 2. Monthly distribution of Chest-CT scans and interpretation errors

\section{Hosted file}

Table 1.pdf available at https://authorea.com/users/393086/articles/506805-how-didradiologists-diagnostic-accuracy-have-changed-in-covid-19-pneumonia-a-single-centreretrospective-study

\section{Hosted file}

Table 2.pdf available at https://authorea.com/users/393086/articles/506805-how-didradiologists-diagnostic-accuracy-have-changed-in-covid-19-pneumonia-a-single-centreretrospective-study

\section{Hosted file}

Table 3.pdf available at https://authorea.com/users/393086/articles/506805-how-didradiologists-diagnostic-accuracy-have-changed-in-covid-19-pneumonia-a-single-centreretrospective-study

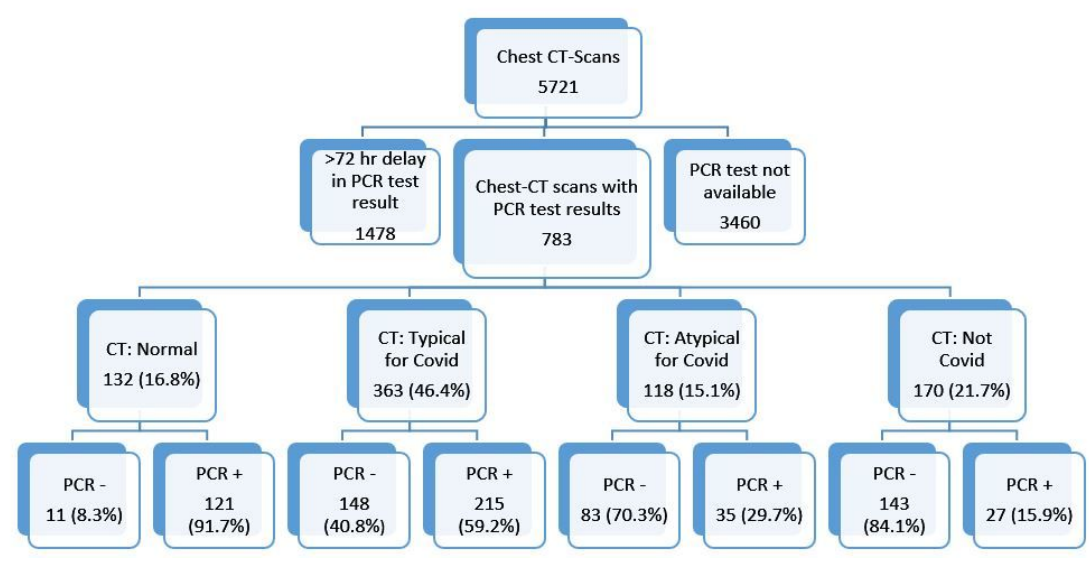




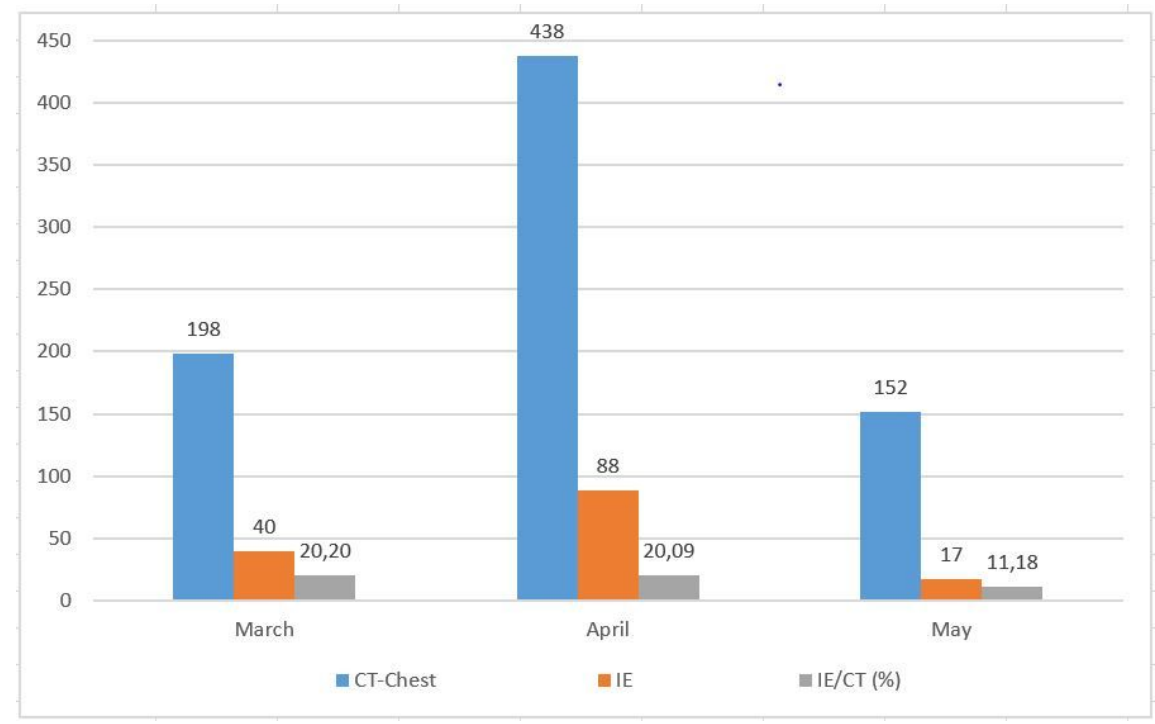

